\title{
Parallel guidewire technique in acute ischemic stroke secondary to carotid artery dissection
}

\author{
Xiongjun He", Liang Zhang", Kaifeng Li, Ming Hu, Hongxing Zhou, Jie Li, Yajie Liu \\ Department of Neurology, Shenzhen Hospital, Southern Medical University, Shenzhen, China \\ Contributions: (I) Conception and design: X He; (II) Administrative support: L Zhang; (III) Provision of study materials or patients: X He, Y Liu; (IV) \\ Collection and assembly of data: K Li, J Li; (V) Data analysis and interpretation: M Hu, H Zhou; (VI) Manuscript writing: All authors; (VII) Final \\ approval of manuscript: All authors. \\ \#These authors contributed equally to this work. \\ Correspondence to: Yajie Liu. Department of Neurology, Shenzhen Hospital of Southern Medical University, No.1333 Xinhu, Baoan District, \\ Shenzhen 518101, China. Email: docliu18@163.com.
}

Background: The aim of the study was to evaluate the influence of endovascular therapy (EVT) on the
acute ischemic stroke (AIS) secondary to carotid artery dissection (CAD).
Methods: This single-center retrospective study enrolled 17 patients admitted with AIS secondary to CAD
from January 2018 to November 2019 in the Department of Neurology, Shenzhen Hospital of Southern
Medical University where patients received EVT with guidewire or parallel guidewire. Outcomes including
postoperative complications were recorded, the prognostic factors of patients were explored, and the
effectiveness of single guidewire versus parallel guidewire on EVT was compared.

Results: Before treatment, the mean National Institute of Health stroke scale (NIHSS) was 14.4 in 10 cases $(58.8 \%)$ of CAD complicated with intracranial artery embolism. All patients underwent EVT, and the success rate of operation was $100 \%$. After all interventions, modified thrombolysis in cerebral ischemia (mTICI) 3 reperfusion was achieved in 14 patients $(82.4 \%$ ), and mTICI 2 b reperfusion was achieved in $3(17.6 \%)$. One patient had cerebral infarction and edema complicated with cerebral hernia, one patient had cerebral hemorrhage, one patient had complicated subarachnoid hemorrhage, and five cases had asymptomatic cerebral hemorrhage. Three months after treatment, 14 cases $(82.3 \%)$ achieved a good clinical outcome (modified Rankin scale, mRS: 0-2). Puncture-to-reperfusion time in the parallel guidewire group was significantly shorter than that of the single guidewire group. However, the differences in NIHSS score, postoperative mTICI, and mRS score between both groups did not reach significance.

Conclusions: CAD patients receiving EVT have a good prognosis, and application of a parallel guidewire can reduce operation time.

Keywords: Carotid artery dissection (CAD); acute ischemic stroke (AIS); revascularization; parallel guidewire

Submitted Oct 14, 2020. Accepted for publication Dec 29, 2020.

doi: 10.21037/apm-20-2168

View this article at: http://dx.doi.org/10.21037/apm-20-2168

\section{Introduction}

Carotid artery dissection (CAD), a known causative factor of acute ischemic stroke (AIS), is characterized by intramural hematoma (IMH) following carotid subintimal tear (1). CAD is not commonly seen in general stroke patients and only accounts for $2-2.5 \%$ of all AIS cases (2), but this disorder is the main cause of stroke in young people, accounting for $10-25 \%$ of all AIS cases (3). Since five major clinical studies noted the efficacy of endovascular therapy (EVT) in acute cerebral infarction (ACI) in 2015, all countries have regarded EVT as the first-level recommendation for ACI due to occlusion of the large vessel. However, there is still no report on the 
operation and treatment guidelines for ACI with large vessel occlusion caused by $\mathrm{CAD}$, especially concerning the dissection of lesions during the operation. In this singlecenter retrospective study, we analyzed the prognosis of 17 patients with AIS secondary to CAD from January 2018 to November 2019 and compared the efficacy of single guide wire and parallel guide wire technique in EVT for CAD. We provide the following articles according to the STROBE reporting checklist (available at http://dx.doi. org/10.21037/apm-20-2168).

\section{Methods}

\section{Clinical information}

Patients with incomplete information were excluded from the study. A total of 17 patients (14 males and 3 females; age, 29-58 years; mean age, 41.8 years) with CAD complicated with AIS were enrolled in this study from January 2018 to November 2019 in the Department of Neurology, Shenzhen Hospital of Southern Medical University. Of the 17 patients, 3 male patients had a history of smoking, 6 had a history of hypertension, 1 had a history of heart disease, 4 had a history hyperlipidemia, 2 had a history of neck massage, and the rest had no obvious risk factors.

\section{Clinical symptoms}

The 17 patients all had acute onset of ischemic stroke symptoms such as dysarthria, central facial and lingual paralysis, hemianopia, hemiplegia, hemidysesthesia, and crossed paralysis. Eleven patients intravenously received thrombolysis with recombinant-tissue plasminogen activator (r-tPA) within 4.5 hours after onset, and with urokinase within 4.5-6 hours. The other patients were assigned with treatment within 24 hours after onset.

\section{Clinical assessment}

Patients who were diagnosed within 6 hours after onset underwent routine head computed tomography (CT). The patients identified over 6 hours after onset were detected through multimodal imaging: head CT angiography (CTA) + CT perfusion (CTP) or magnetic resonance imaging (MRI) diffusion-weighted imaging (DWI) + arterial spin labeling (ASL) + magnetic resonance angiography (MRA) to assess ischemic penumbra and great vessels and to determine the presence of occlusion and mismatched areas. For the judgment of the nature of carotid artery occlusion, all cases in this center are interpreted by two neuroimaging departments and neurointerventional doctors. The imaging diagnostic criteria for arterial dissection include the following: (I) fusiform or irregular tumor-like expansion of non-branched blood vessels; (II) long, filamentous, and irregular stenosis, with one or more of the following manifestations: double lumen, Intramural hematoma, endometrial valve, rapid morphological changes, focal stenosis and dilation (bead-like changes); or (III) recanalization of non-branched vessels accompanied by fusiform or irregular aneurysm-like dilatation. These standards are mainly based on conventional angiography, CTA or MRA imaging results.

\section{Treatments}

Patients detected within 6 hours after onset with National Institute of Health stroke scale (NIHSS) scores $>6$ and Alberta stroke program early CT (ASPECT) scores $>6$ underwent EVT with bridging intravenous thrombolysis, in strict accordance with standards of EVT for AIS. Those detected after more than 6 hours from onset with ASPECT or pc-ASPECT $>6$, or NIHSS score $>6$, underwent multimodal imaging assessment. Patients with an obvious mismatch in the penumbral areas, according to the DAWN or DIFFUSE3 trial standards, were given EVT. All operations received informed consent from the patients' family members, and all patients participated in the 13th National Five-Year Plan Registered Study. All procedures performed in the studies involving human participants were in accordance with the Helsinki Declaration (as revised in 2013). This study was approved by the Clinical Research Ethics Committee of Shenzhen Hospital of Southern Medical University. All operations received informed consent from the patients' family members. Source data of this study are available from the corresponding author on reasonable request.

\section{Surgical procedure}

Most of the patients undergoing surgery received local anesthesia and sedation, and only patients (29.4\%) received general anesthesia through the trachea cannula when sedation failed to control these patients.

Aortography and intracranial angiography were initially performed with a $5-\mathrm{Fr}$ pig tail catheter through the 8 - Fr shuttle guiding sheath to evaluate vascular occlusion and 
general compensation. For patients with tortuous arcus aortae, an 8-Fr NeuroMax (penumber) was inserted as a guiding catheter under the guidance of a 5 -Fr multifunction tube (Cordis) and the guide wire to locate vascular lesions and observe the morphology. Then, in one group, we inserted an SL-10 microcatheter (Stryer) or XT-27 microcatheter (Stryker) across the lesion using a $300 \mathrm{~cm}$ single guide wire (Synchro or Transend 0.014, Stryker) until the tip had passed sufficiently into the lesion. In another group, a parallel guide wire was used to pass through the dissected lesion. We tried two times to first have a Synchro or Transend guide wire pass through false lumen, but this still failed, and the guide wire was left in the lumen to block the pathway. The second guide wire was utilized to access true lumen from different angles for cross artery dissection, and was left in the distal normal blood vessels. A microcatheter was inserted along the second guide wire across the dissection lesion and placed in the distal vascular cavity, which was followed by the withdrawal of the guide wire. After the microcatheter was confirmed to be located in the true vascular cavity, the guide wire in the false lumen was removed.

For CAD complicated with distal thrombosis, different strategies for two distinct situations were used. (I) If the thrombotic load of the proximal dissection was not large or did not affect the passage of the intermediate catheter or aspiration catheter, the intracranial lesions was first treated, following insertion of the middle catheter or the ACE aspiration catheter across the dissected lesion to the distal occlusion site, along the micro-guide wire and microcatheter. A direct aspiration first pass technique (ADAPT) was used for aspiration. If the intracranial thrombus load was large, the Solumbra technique with a stent retriever was used to remove the embolus. After recanalizing the distal blood vessels, the protective umbrella (Spider, Medtronic) was added to petrous bone segment along the aspiration catheter. The intermediate catheter or the aspiration catheter was withdrawn into the guide tube. We used the Wallstent stent (Boston Scientific) to apply the $\mathrm{CAD}$ along the protective umbrella micro-guide wire. (II) If the cervical dissection affected the passage of the intermediate catheter or the suction catheter, or if a large thrombus load was present in the proximal treatment locations, the proximal dissection was treated first, with the possibility that the thrombus could fall off again being considered. A Wallstent stent was directly applied to the carotid artery dissection along the micro-guide wire. Then, the intermediate catheter or the ACE aspiration catheter
(Penumber) was passed through the proximal stent and remained on the distal occlusion site, with ADAPT being used to ensure the opening. If the intracranial blood vessel thrombosis load was large, the Solumbra technique with stent was used to remove the embolus.

Technical results of recanalizing therapies were scored by the modified thrombolysis in cerebral ischemia (mTICI) scale. mTICI $2 \mathrm{~b}$ and mTICI 3 are conventionally subsumed under successful recanalization. Before stent implantation, patients were administered with an intravenous injection of $6 \mathrm{~mL}$ of tirofiban and $5 \mathrm{~mL} / \mathrm{h}$ of maintenance fluid. Energy spectrum CT performed immediately after surgery to identify bleeding or contrast agent retention, and exclude bleeding patients who were given continuous pumping of tirofiban for 24 hours and then replaced with dual antiplatelet therapy (DAPT, aspirin and Plavix), for infarctions with a small amount of blood leakage Patients were treated with monoclonal Antiplatelet Therapy (Polivix). If the bleeding is heavy, antiplatelet therapy is not given. After 3 months, the postoperative treatment of DAPT was changed to monoclonal antiplatelet therapy.

\section{Clinical characteristics and outcome assessment}

The primary outcome was functional independence at 90 days. Functional independence was defined as an mRS score of $0-2$. The mRS score was assessed by a neurologist blinded to the treatment detail via telephone interview. The secondary outcomes were successful recanalization at the end of the procedure and puncture to recanalization time. Successful recanalization was defined $\mathrm{mTICI} \geq 2 \mathrm{~b}$. The safety outcome was symptomatic hemorrhage (sICH) within 24 hours. sICH was evaluated on CT and regarded as symptomatic if the patient's neurologic symptoms deteriorated with an increase of $\geq 4$ on the NIHSS.

\section{Statistical analysis}

Baseline, clinical and procedural characteristics, outcomes were compared between the single guide wire group and the parallel guide wire group. The Mann-Whitney U test, $\chi^{2}$ test, and Fisher exact test were used for comparison. A value of $\mathrm{P}<0.05$ was considered significant.

\section{Clinical scenarios}

\section{Case 1}

A 54-year-old man was admitted to the hospital at 19:40, 

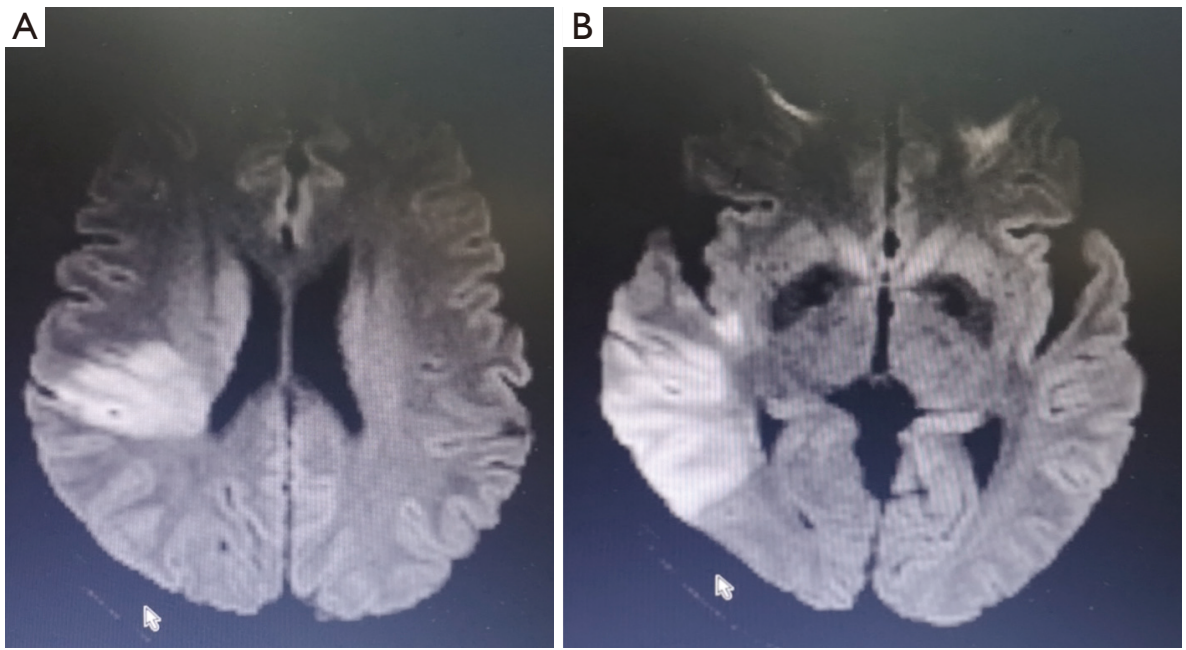

Figure 1 MRI imaging of the right temporal lobe (A) and the radiated coronary area (B) demonstrates cerebral infarction. MRI, magnetic resonance imaging.

September 23, 2019 due to left limb weakness and slurred speech. His symptoms appeared abruptly 8 hours earlier in the workplace without obvious cause. He could not walk on his own and had slurred speech, but no relief after rest. He was then rushed to Shenzhen Baoan Traditional Chinese Medicine Hospital. At that time, head CT indicated no brain bleeding. Intravenous thrombolysis with $1 \times 10^{6} \mathrm{IU}$ of urokinase was administered to the patient at 16:30. After thrombolysis at 17:00, slurred speech and weakness of the left limb were temporarily improved. About 20 minutes after the patient completed the head MR scan + DWI + MRA, the left limb fell limp again. The head MRI showed acute brain infarction in the right temporal lobe and a radiated coronary area (Figure 1). The condition worsened, and the patient was transferred to the emergency department of our hospital. He is a professional driver and has neck pain upon neck massage. In the emergency department, he had a blood pressure of $140 / 75 \mathrm{mmHg}$, a clear mind, slurred speech, no drooping of either eyelid, and a diameter in his pupils of 3.0 $\mathrm{mm}$. His eyes had no nystagmus, his mouth was angled to the right, his uvula was centered, and his soft palate could be lifted. The muscle strength of the right limb was grade 5, the left upper limb was grade 1 , and the left lower limb was grade 3 . The pain and touch sensation on the left side were slightly weakened. The right side of his body could perform coordinated movement. His neck was soft, and the Gram and Brinell signs were negative. The NIHSS score was 9, and the ASPECT score was 7.
Intraoperative angiography confirmed that the initiation of the right internal carotid artery was occluded, and that the left internal carotid artery was partially compensating for this with anterior traffic to the right middle cerebral artery area (Figure 2). The 0.014 in $300 \mathrm{~mm}$ Synchro microguide wire was selected to pass through the $\mathrm{CAD}$, but this failed (Figure $3 A$ ) and remained in the false cavity. Another $0.014300 \mathrm{~mm}$ Transend micro-guide wire successfully crossed the dissection site (Figure 3B,C), and the XT microcatheter was inserted along the Transend microguide wire to the $\mathrm{C} 2$ section of the internal carotid artery, followed by withdrawal of the micro-guide wire. Microcatheter angiography confirmed that it was located in the true lumen of the blood vessel (Figure 4A), and that no occlusion in the distal middle cerebral artery was present (Figure 4B). The $0.014300 \mathrm{~mm}$ Transend micro-guide wire was placed in the $\mathrm{C} 2$ section, and the microcatheter is removed. The Wallstent $(7 \times 50 \mathrm{~mm})$ self-expanding stent was placed in the cervical dissection and applied to dissection. The blood vessel was formed (Figure 4C,D) and the mTICI score returned to grade 3 .

The next day after operation, head CT indicated no hemorrhage (Figure $5 A, B$ ). The patient was given tirofiban for 24 hours, which was then was replaced with BsAb. Three months after surgery, the angiography suggested that the right carotid artery showed smooth blood circulation (Figure $5 C, D$ ), and the patient could speak clearly. The muscle strength had returned to level 5 , and 

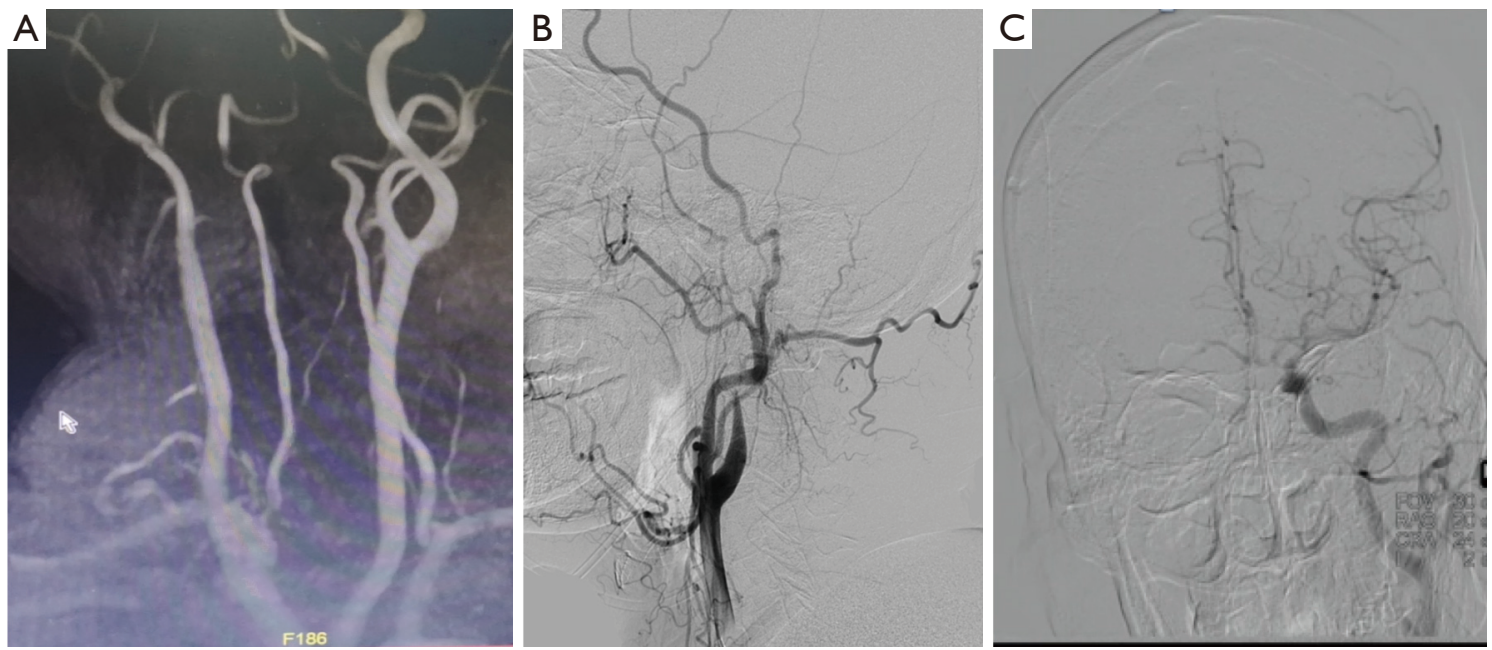

Figure 2 MRA imaging indicates occlusion of the right internal carotid artery (A). Angiography shows the occlusion in the start of the right internal carotid artery of a rat tail (B) and that the anterior communicating artery before the left internal carotid artery is open, compensating for the right side anterior cerebral artery (C). MRA, magnetic resonance angiography.
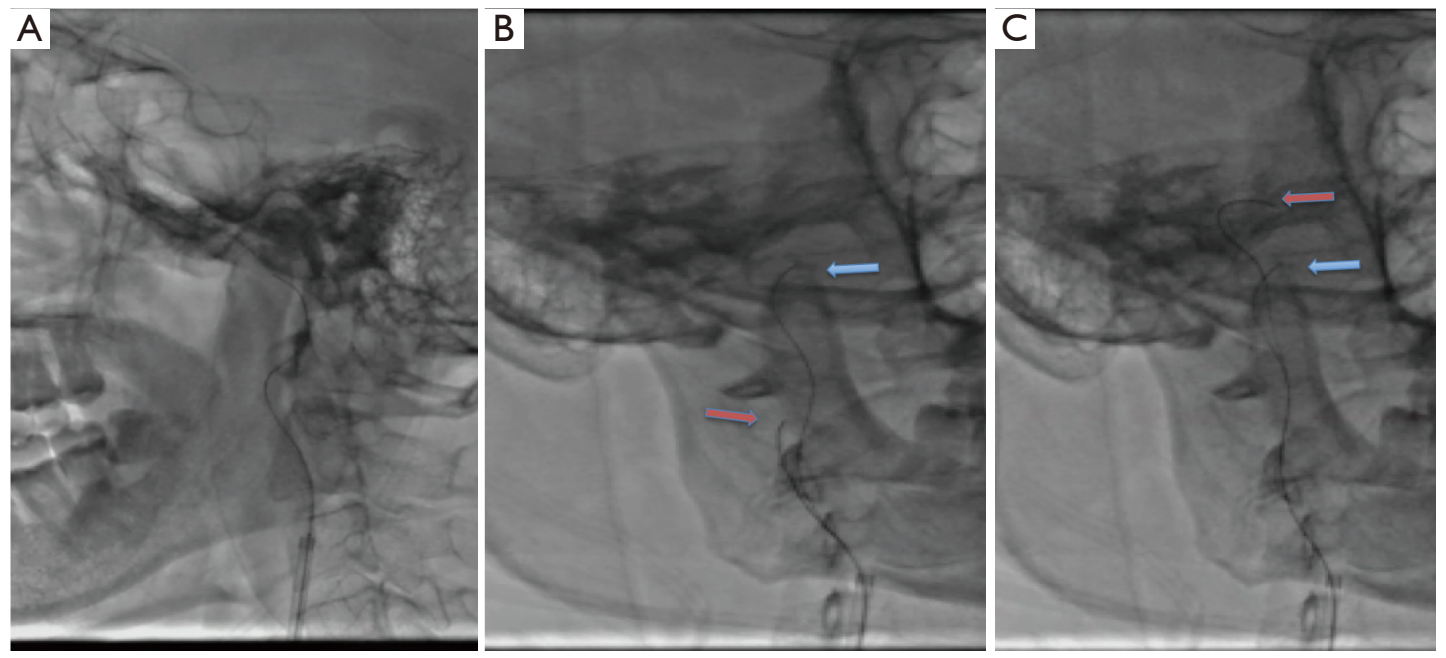

Figure 3 The micro-guide wire barely passes through the neck (A), the guide wire (blue arrow) is fixed in the false cavity (B), and another guide wire (red arrow) is re-attached and passes through the distal true cavity (C).

the mRS score was 0 .

\section{Results}

In the postoperative effect observation and follow-up of 17 patients, hypertension appeared in 6 cases, smoking history in 3 cases, hyperlipidemia in 4 cases, and clear neck trauma or massage history in 2 cases. The preoperative average NIHSS score of patients was 14.4 points, and the
ASPECT score was 8.5 points. Eleven patients detected within 6 hours after onset were given thrombolysis. Of the 17 patients, the success rate of the opening of an occluded coronary vessel was $100 \%$. The postoperative recanalization was evaluated according to the mTICI classification, where anterograde blood flow of mTICI $2 \mathrm{~b}$ was considered partial recanalization, and mTICI 3 was considered complete recanalization. Of the 17 patients, 14 achieved mTICI 3 reperfusion, and 3 achieved mTICI $2 \mathrm{~b}$ reperfusion. 



Figure 4 Micro-catheter angiography imaging reveals that the micro-guide wire is located in the true cavity of the blood vessel (A, blue arrow) and that the distal blood vessel is unobstructed (B, blue arrow). A Wallstent $(7 \times 50 \mathrm{~mm})$ stent is implanted well and dissection repair is normal (C, blue arrow), and the intracranial branches are visible (D).

Meanwhile, 5 patients developed asymptomatic brain hemorrhage, and 1 had symptomatic cerebral hemorrhage, The hemorrhage transformation after cerebral infarction is divided into HI type and $\mathrm{PH}$ type. HI (hemorrhagic infarction), which means hemorrhagic cerebral infarction, refers to small spotted hemorrhage with unclear borders; PH (parenchymal hemorrhage), which means cerebral parenchymal hemorrhage, refers to a mass hematoma with clear borders, with space-occupying effect. The two types can be divided into HI1 and HI2 PH1 and PH2 according to the degree of bleeding. The significance of the classification is that it is closely related to the prognosis. The hematoma type, which is the $\mathrm{PH}$ type, has a worse prognosis, and the $\mathrm{PH} 2$ fatality rate reaches $50 \%$. Suction was performed to these patients during treatment. Three months later, the number of cases with an mRS score of $0-2$ was $14(82.3 \%)$.

Of the 17 patients (see Tables 1,2 for details), 7 patients $(41.2 \%)$ presented with middle cerebral artery occlusion, and 4 patients $(23.5 \%)$ with internal carotid artery occlusion. Intraoperative digital subtraction angiography (DSA) assessment of ASITN/SIR collateral circulation classification revealed the following: 11 grade 2 cases, 4 grade 1 cases, and 2 grade 0 cases. The EVT strategy was performed as described above. A single guide wire was used in 10 cases to pass the dissection lesion, and a parallel guide wire was used in 7 cases. Stent angioplasty was first performed in 9 patients where a self-expanding stent 




Figure 5 Re-examination of head CT immediately after surgery reveals no intracranial hemorrhage (A), and the lesions are same as those before the operation (B). At 3-month reexamination, the stent position is good without restenosis, and the dissection has healed (C,D).

was placed in the dissection to establish an opening. The guide tube and the intermediate catheter were then passed through the stent to the distal end to remove the thrombus. Of these patients, a thrombus removal stent or aspiration catheter was used to open the distal occluded blood vessel in 5 cases, and in 4 cases the opening of the CAD allowed the distal thrombosis to canalize. The other 8 cases underwent thrombectomy firstly via insertion of an ACE aspiration catheter into the middle cerebral artery occlusion and internal carotid artery occlusion, as the catheter was easy to pass through the dissection. Of these 8 cases, 4 received Wallstent self-expanding stent implantation at the later stage, and the others were not further treated because their CAD did not affect the blood flow, their clinical symptoms were significantly relieved, and the vessel was not occluded as indicated by CT scans.

The comparison of CAD with a single guide wire to that with a parallel guide wire (see Table 3) revealed no differences in risk factors, age, or ASPECT score. Of note, the single guide wire group had a high preoperative NIHSS score and thrombolysis rate. With time from admission to puncture being insignificant between the groups, the application of parallel guide wire shortened the time from puncture to first recanalization, and from puncture to complete recanalization. The difference between both groups in postoperative mTICI blood flow and 3-month prognosis did not reach significance, as the rate of good prognosis was over $80 \%$ in both groups. 
Table 1 Clinical data of 17 patients with acute ischemic stroke (AIS)

\begin{tabular}{|c|c|}
\hline Variable & $\begin{array}{c}\text { Number of patients } \\
\text { (mean or ratio) }\end{array}$ \\
\hline Age (year) & 41.8 \\
\hline Gender (male) & $14(82.3 \%)$ \\
\hline Hypertension & $6(35.3 \%)$ \\
\hline Smoking & $3(17.6 \%)$ \\
\hline Hyperlipidemia & $4(23.5 \%)$ \\
\hline History of trauma & $2(11.8 \%)$ \\
\hline Preoperative NIHSS score & 14.4 \\
\hline ASPECT score & 8.5 \\
\hline \multicolumn{2}{|l|}{ ASITN/SIR collateral scale } \\
\hline Grade 2 & $11(64.7 \%)$ \\
\hline Grade 1 & $4(23.5 \%)$ \\
\hline Grade 0 & 2 (11.8\%) \\
\hline Thrombolysis & $11(64.7 \%)$ \\
\hline$r$-tPA & 9 (52.9\%) \\
\hline Urokinase & $2(11.8 \%)$ \\
\hline \multicolumn{2}{|l|}{ Anesthesia } \\
\hline Local anesthesia & $12(70.6 \%)$ \\
\hline General anesthesia & $5(29.4 \%)$ \\
\hline
\end{tabular}

NIHSS score: National Institute of Health stroke scale. The higher the score, the more serious the condition. $\mathrm{mTICl}$ : modified treatment in cerebral infarction (mTICl) score (4); grade 0: no perfusion; grade 1: little blood passing through the occlusion segment, with little or no reperfusion; grade 2a: antegrade reperfusion of less than half of the target artery previously in the ischemic territory; grade $2 \mathrm{~b}$ : antegrade reperfusion of more than half of the previously target artery ischemic territory; grade 3: complete antegrade reperfusion of the downstream artery ischemic territory. The American Society of Intervention and Therapeutic Neuroradiology/Society of Interventional Radiology (ASITN/SIR) collateral compensation scale is a 5 -point scale; grade 0 : no collaterals visible to the ischemic site; grade 1: slow collateral compensation to the periphery of the ischemic site with persistence of some of the defect; grade 2: rapid collaterals compensation to the periphery of the ischemic site with persistence of some of the defect; grade 3: collaterals compensation with slow but complete angiographic blood flow of the ischemic bed by the late venous phase; grade 4: complete and rapid collateral compensation blood flow to the vascular bed in the entire ischemic territory.
Table 2 Lesions and operation results

\begin{tabular}{lc}
\hline Surgical factors & $\begin{array}{c}\text { Number of cases } \\
\text { (mean or ratio) }\end{array}$ \\
\hline Tandem occlusion & $11(64.7 \%)$ \\
Middle cerebral artery & $7(41.2 \%)$ \\
Intracranial internal carotid artery & $4(23.5 \%)$ \\
Preferred strategy & \\
Angioplasty and stenting & $9(52.9 \%)$ \\
Stent thrombectomy & $2(11.8 \%)$ \\
Suction & $6(35.3 \%)$ \\
Remedy & \\
Angioplasty and stenting & $4(23.5 \%)$ \\
Suction & $5(29.4 \%)$ \\
Puncture-recanalization time (min) & 142.47 \\
mTICl grade & $14(82.4 \%)$ \\
mTICI 3 & $14(82.4 \%)$ \\
mTICl 2b & $3(17.6 \%)$ \\
Ilb/Illa use & $12(35.6 \%)$ \\
All intracranial hemorrhage & \\
\hline montomatic intracranial hemorrhage & \\
\hline
\end{tabular}

Modified Rankin scale (mRA): 0-5 points, the higher the branch, the worse the prognosis. 0: no symptoms; 1 : no significant disability despite symptoms, able to carry out all usual duties and activities; 2: slight disability, unable to carry out all previous activities but not requiring assistance; 3: moderate disability, requiring some help, but able to walk without assistance; 4: moderately severe disability, can walk with assistance but unable to attend to own needs without assistance; 5: severe disability, bedridden, incontinent, and requiring constant nursing care and attention. $\mathrm{mTICl}$, modified thrombolysis in cerebral ischemia; MRA, magnetic resonance angiography. 
Table 3 Comparison of the single guide wire and the parallel guide wire

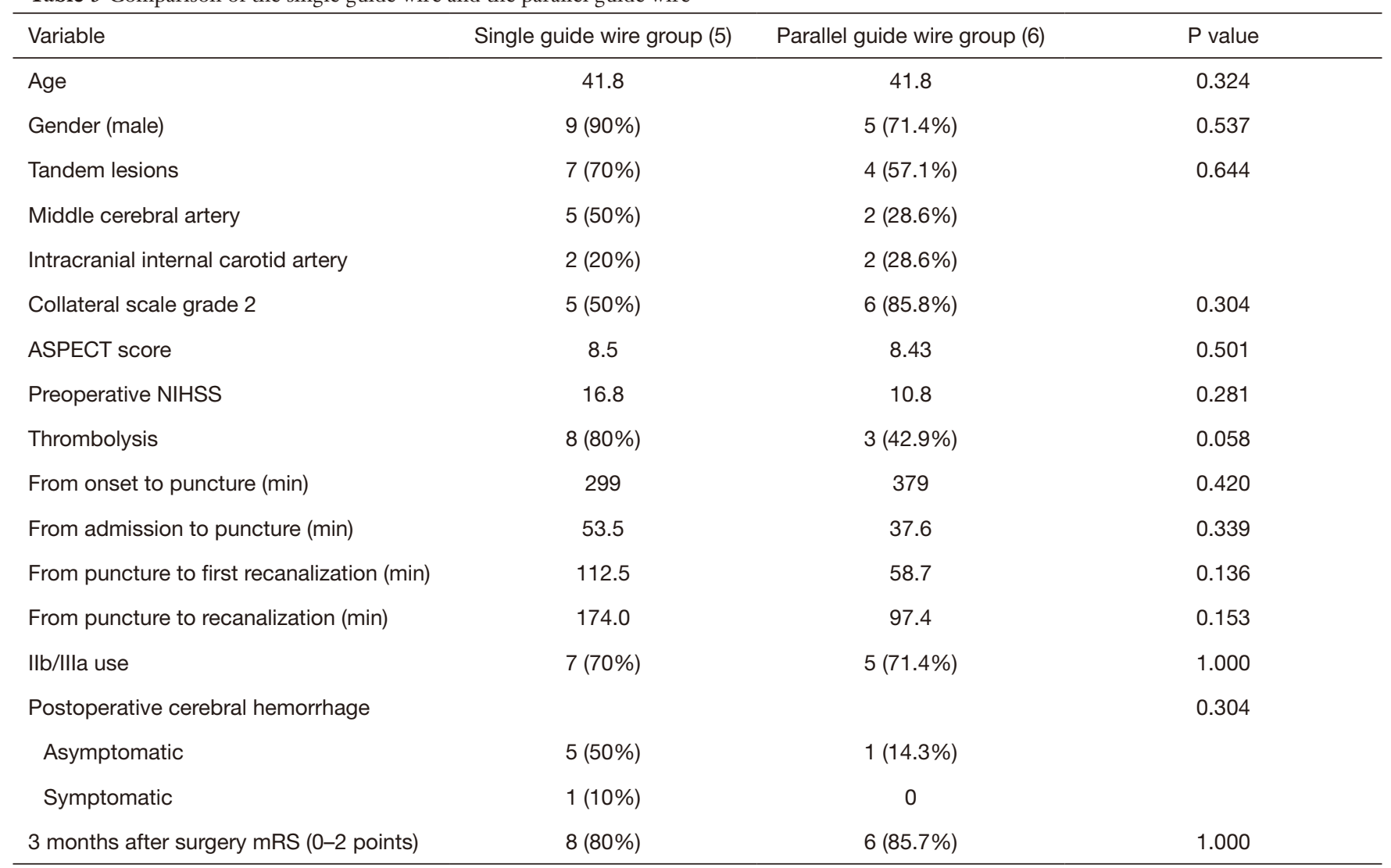

The ASPECT is a 10-point scale, where points are lost for each region affected. The lower the score, the more infarcted parts. ASPECT, Alberta stroke program early CT; NIHSS, National Institute of Health stroke scale; mRS, modified Rankin scale.

After discharge, the telephone follow-up indicated that patients' symptoms had improved significantly. After successful opening of the CAD, patients did not have an ischemic attack in the ipsilateral carotid artery blood supply area. All patients were rechecked with CTA or cerebrovascular angiography 3 months later, and the results indicated that the original occluded carotid artery was unobstructed, with the distal blood vessel having developed well, with no collateral circulation. After surgery, 13 patients with carotid artery stents were given antiplatelet drugs, lipid lowering therapy, and plaque stabilization. They orally ingested statins (Lipitor 20-60 mg once a day), $75 \mathrm{mg}$ of clopidogrel bisulfate, and aspirin in combination with $100 \mathrm{mg}$ of BsAb or monoclonal antibody for 3 months. After 3 months of the reexamination of blood vessels, treatment switched to monoclonal antibody; 4 patients without stent placement took anticoagulation therapy after discharge, and 3 months later, reexamination of angiography indicated no stenosis of the blood vessel at the stent.

\section{Discussion}

The annual incidence of CAD is approximately 10.4\% (7). Transcranial Doppler (TCD) ultrasound can detect cerebral micro-embolisms in $46 \%$ to $59 \%$ of patients with $\mathrm{CAD}$, which suggests that patients with $\mathrm{CAD}$ are prone to cerebral embolisms in the arterial supply $(6,8)$. In addition, $20 \%$ of patients with CAD complicated by intracranial vascular occlusion also have severe internal carotid artery stenosis or even complete occlusion (9). These patients have a low recanalization rate upon intravenous thrombolysis with rt-PA (only $31 \%$ ). Most patients with middle cerebral artery occlusion have a poor prognosis $(5,10)$. There were 11 patients in our study who received intravenous thrombolysis but whose blood vessels were not recanalized. The subsequent bridging mechanical thrombectomy was used to recanalize the blood vessels.

One meta-analysis compared the efficacy of intravenous thrombolysis and intravascular interventional therapy 
in patients with ACI secondary to CAD, revealing that intravascular interventional therapy alone achieved better outcomes than intravenous thrombolysis alone, but there was no significant difference in cerebral hemorrhage, mortality, or stroke recurrence (11). In another metaanalysis, 6 clinical data of ACI secondary to CAD were selected, comprising 193 patients who received interventional thrombectomy and 59 who received medical treatment. After thrombectomy, 74\% of patients were restored to grade $2 \mathrm{~b}-3$ blood flow. The interventional thrombectomy group (62.9\%, 95\% CI: 55.8-69.5\%) obtained better prognosis at 3 months (mRS 0-2) relative to the drug treatment group (41.5\%, 95\% CI: $29.0-55.1 \%$; $\mathrm{P}=0.006)$. The 90 -day mortality upon interventional thrombectomy was similar to that obtained with drug treatment $(8.6 \%$ and $6.3 \%)$, and the difference in the incidence of symptomatic intracranial hemorrhage (sICH) was not significant $(5.9 \%$ vs. $4.2 \%, \mathrm{P}=0.60)(12)$. In our study, the success rate of operation in the 17 patients was $100 \%$. After all interventions, 1 patient had complicated subarachnoid hemorrhage, and 5 had asymptomatic cerebral hemorrhage. Three months later, $\mathrm{mRS}$ scores were improved (0-2) in 14 cases, which was higher than the rate of improvement of the Lin (11) and Dmytriw (12) study. This might be due to the fact that patients in our study had good collateral compensation. However, a 15-year follow-up study on ACI due to CAD in multiple centers revealed that in 109 total patients, 24 received EVT (bridging treatment), 38 received intravenous thrombolysis, and 47 received no vascular recanalization treatment. Patients with EVT had a higher rate of vascular recanalization within 24 hours, and were more vulnerable to non-symptomatic bleeding when compared to patients given control treatment $(\mathrm{P}=0.026)$. There was no difference in sICH and mortality within 7 days between the three groups. The rate of good clinical prognosis achieved at 3 months did not differ between the different subgroups (intravascular treatment: intravenous thrombolysis $\mathrm{P}=0.407$; $\mathrm{EVT}$ : no recanalization treatment $\mathrm{P}=0.580)(13)$.

CAD leads to acute occlusion, and is often complicated with intracranial artery embolism. At present, no research exists specifically focusing on the EVT of ACI caused by CAD. Surgical methods for tandem lesions include treating the proximal dissection lesion first, followed by the opening of the distal occluded vessel, and vice versa. A study by Stampfl et al. (14) enrolled 24 patients with tandem occlusion of the internal carotid artery and middle cerebral artery, where patients were administrated with middle cerebral artery stent embolization and internal carotid artery stent implantation. Among these patients, $62.50 \%(15 / 24)$ of patients achieved recanalization. (TICI grade 2 b-3), but only $29.17 \%$ (7/24) of patients had a good clinical prognosis 3 months after operation (mRS score $\leq 2$ points). For tandem occlusion, some scholars suggest that carotid artery disease should be routinely treated before the distal occlusion, so that the proximal and distal recanalization rates can exceed $84 \%$ and $33 \%$, respectively (15-19). The advantages of first restoring carotid artery blood flow are improving the safety of entering the distal disease, the removal rate of distal thrombus, and the simultaneous increase of arterial rt-PA activity due to the increase in anterograde blood flow, which in turn reduces the risk of distal re-occlusion and ischemia (16). Regardless of the surgical method, the main purpose is to open the occluded blood vessels in a timely and effective manner and to quickly restore brain tissue perfusion. Given this, the EVT strategy involves passing the proximal dissection lesion. In this study, we used a single guide wire to pass the dissection lesion in 10 cases, and a parallel guide wire in 7 cases. There were no differences in risk factors, age, or ASPECT scores between both guide wire types. The single guide wire group had a high preoperative NIHSS score and thrombolysis rate. As the time from admission to puncture was similar in both groups, it took less time to complete the recanalization in the guide wire group. The difference between both groups in postoperative mTICI blood flow and 3-month prognosis did not reach significance, as their rate of good prognosis exceeded $80 \%$.

Despite these findings, there remain some limitations to our study which should be noted. First, the diagnosis of the cause of vascular occlusion mainly relies on the comprehensive judgment of two neuro-interventional radiologists based on the patient's clinical data and imaging data. There is a lack of a unified gold standard, and there might have been a certain selection bias present. Furthermore, this study is a single-center retrospective study with a small sample size. Some patients were excluded due to missing data, resulting in possible selection bias in this study.

\section{Conclusions}

Our study indicates that CAD patients receiving EVT may be reasonable, and application of a parallel guidewire will reduce operation time. A large prospective study is necessary for confirmation because of the small sample size 
of this study.

\section{Acknowledgments}

Funding: This study was supported by the Sanming Project of Medicine in Shenzhen (SZSM201812047) and the Municipal Key Clinical Department in Shenzhen (SZXK074).

\section{Footnote}

Reporting Checklist: The authors have completed the STROBE reporting checklist. Available at http://dx.doi. org/10.21037/apm-20-2168

Data Sharing Statement: Available at http://dx.doi. org/10.21037/apm-20-2168

Conflicts of Interest: All authors have completed the ICMJE uniform disclosure form (available at http://dx.doi. org/10.21037/apm-20-2168). The authors have no conflicts of interest to declare.

Ethical Statement: The authors are accountable for all aspects of the work in ensuring that questions related to the accuracy or integrity of any part of the work are appropriately investigated and resolved. All procedures performed in the studies involving human participants were in accordance with the Helsinki Declaration (as revised in 2013). This study was approved by the Clinical Research Ethics Committee of the Shenzhen Hospital of Southern Medical University. All operations received informed consent from the patients' family members.

Open Access Statement: This is an Open Access article distributed in accordance with the Creative Commons Attribution-NonCommercial-NoDerivs 4.0 International License (CC BY-NC-ND 4.0), which permits the noncommercial replication and distribution of the article with the strict proviso that no changes or edits are made and the original work is properly cited (including links to both the formal publication through the relevant DOI and the license). See: https://creativecommons.org/licenses/by-nc-nd/4.0/.

\section{References}

1. Schievink WI, Mokri B, O'Fallon WM. Recurrent spontaneous cervical-artery dissection. N Engl J Med
1994;330:393-7.

2. Bassetti C, Carruzzo A, Sturzenegger M, et al. Recurrence of cervical artery dissection. A prospective study of 81 patients. Stroke 1996;27:1804-7.

3. Mohr JP, Thompson JL, Lazar RM, et al. A comparison of warfarin and aspirin for the prevention of recurrent ischemic stroke. N Engl J Med 2001;345:1444-51.

4. Zaidat OO, Yoo AJ, Khatri P, et al. Recommendations on angiographic revascularization grading standards for acute ischemic stroke: a consensus statement. Stroke 2013;44:2650-63.

5. Rubiera M, Ribo M, Delgado-Mederos R, et al. Tandem internal carotid artery/middle cerebral artery occlusion: an independent predictor of poor outcome after systemic thrombolysis. Stroke 2006;37:2301-5.

6. Srinivasan J, Newell DW, Sturzenegger M, et al. Transcranial Doppler in the evaluation of internal carotid artery dissection. Stroke 1996;27:1226-30.

7. Beletsky V, Nadareishvili Z, Lynch J, et al. Cervical arterial dissection: time for a therapeutic trial? Stroke 2003;34:2856-60.

8. Lucas C, Moulin T, Deplanque D, et al. Stroke patterns of internal carotid artery dissection in 40 patients. Stroke 1998;29:2646-8.

9. Grau AJ, Weimar C, Buggle F, et al. Risk factors, outcome, and treatment in subtypes of ischemic stroke: the German stroke data bank. Stroke 2001;32:2559-66.

10. Tallarita T, Lanzino G, Rabinstein AA. Carotid intervention in acute stroke. Perspect Vasc Surg Endovasc Ther 2010;22:49-57.

11. Lin J, Liang Y, Lin J. Endovascular therapy versus intravenous thrombolysis in cervical artery dissectionrelated ischemic stroke: a meta-analysis. J Neurol 2020;267:1585-93.

12. Dmytriw AA, Phan K, Maingard J, et al. Endovascular thrombectomy for tandem acute ischemic stroke associated with cervical artery dissection: a systematic review and meta-analysis. Neuroradiology 2020;62:861-6.

13. Bernardo F, Nannoni S, Strambo D, et al. Efficacy and safety of endovascular treatment in acute ischemic stroke due to cervical artery dissection: A 15-year consecutive case series. Int J Stroke 2019;14:381-9.

14. Stampfl S, Ringleb PA, Mohlenbruch M, et al. Emergency cervical internal carotid artery stenting in combination with intracranial thrombectomy in acute stroke. AJNR Am J Neuroradiol 2014;35:741-6.

15. Jovin TG, Gupta R, Uchino K, et al. Emergent stenting of extracranial internal carotid artery occlusion in acute stroke 
has a high revascularization rate. Stroke 2005;36:2426-30.

16. Cohen JE, Gomori JM, Rajz G, et al. Extracranial carotid artery stenting followed by intracranial stentbased thrombectomy for acute tandem occlusive disease. J Neurointerv Surg 2015;7:412-7.

17. Nedeltchev K, Brekenfeld C, Remonda L, et al. Internal carotid artery stent implantation in 25 patients with acute stroke: preliminary results. Radiology 2005;237:1029-37.

18. Dabitz R, Triebe S, Leppmeier U, et al. Percutaneous recanalization of acute internal carotid artery occlusions

Cite this article as: $\mathrm{He} \mathrm{X}$, Zhang $\mathrm{L}, \mathrm{Li} \mathrm{K}, \mathrm{Hu} \mathrm{M}$, Zhou H, Li J, Liu Y. Parallel guidewire technique in acute ischemic stroke secondary to carotid artery dissection. Ann Palliat Med 2021;10(1):266-277. doi: 10.21037/apm-20-2168 in patients with severe stroke. Cardiovasc Intervent Radiol 2007;30:34-41.

19. Lavallee PC, Mazighi M, Saint-Maurice JP, et al. Stentassisted endovascular thrombolysis versus intravenous thrombolysis in internal carotid artery dissection with tandem internal carotid and middle cerebral artery occlusion. Stroke 2007;38:2270-4.

(English Language Editor: J. Gray) 